$\mathrm{p}=0.014)$. NLR, alone, was not predictive of MACE at either 30 days or 12 months $(p=N S)$. Of the two-biomarker combinations, enhanced platelet reactivity and raised hs-CRP together was the strongest predictor of MACE at 30 days (HR 2.32 [95\% CI 1.71-3.13], p<0.001) and 12 months (HR 2.31 [95\% CI 1.71-3.11], p<0.001). The combination of all three biomarkers, however, was the strongest overall predictor of MACE at 30 days (HR 2.58 [95\% CI 1.783.75], $\mathrm{p}<0.001$ ) and 12 months (HR 2.61 [95\% CI 1.803.80], $\mathrm{p}<0.001$ ) (Figure 1).

Conclusion In patients with STEMI undergoing PPCI, although individually, each biomarker was poorly predictive, in combination provide significant additive predictive value for the occurrence of MACE at 30 days and 12 months.

Conflict of Interest None

\section{THE DERIVATION OF TROPONIN-ONLY MANCHESTER ACUTE CORONARY SYNDROMES (T-MACS) DECISION AID WITH ALERE POINT OF CARE FOR RULING OUT ACS IN PATIENTS WITH CHEST PAIN PRESENTED TO EMERGENCY DEPARTMENT}

${ }^{1}$ Abdulrhman Alghamdi*, ${ }^{2}$ Charles Reynard, ${ }^{1}$ Richard Body. ${ }^{1}$ The university of Manchester; ${ }^{2}$ Manchester University NHS Foundation Trust

\subsection{6/heartjnl-2019-BCS.153}

Introduction The Troponin-only Manchester Acute Coronary Syndromes (T-MACS) decision aid is a prospectively validated tool to rule-in and rule-out acute coronary syndromes (ACS) using a single blood test in the Emergency Department (ED). T-MACS has been validated with laboratory-based cardiac troponin (cTn) assays. The rapid turnaround time of point of care (POC) cTn assays has great potential advantages for busy EDs. We therefore aimed to evaluate the diagnostic accuracy of T-MACS using a contemporary POC cTn assay.

Method We conducted a secondary analysis of the Bedside Evaluation of Sensitive Troponin (BEST) study, a prospective diagnostic accuracy study at 14 UK centres. We included adults with chest pain of $<12$ hours duration, which the treating physician suspected to be ACS. We excluded patients with ST elevation myocardial infarction; patients with another medical condition requiring hospitalization; and patients lacking capacity to provide written informed consent. Blood was drawn on arrival and 3 hours later for POC cTnI using the Alere Cardiac Triage 3 assay (99th percentile $0.02 \mathrm{ng} / \mathrm{ml}$ ). The accuracy of T-MACS was calculated using (a) the result on arrival alone (T0 analysis); and (b) the maximum POC cTn result based on presentation and 3-hour samples (T3 analysis).

The target condition was acute myocardial infarction (AMI), which was adjudicated by two investigators based on serial cTn testing over 3-6 hours. We also followed patients for major adverse cardiac events (MACE; including death, AMI or coronary revascularization) at 30 days.

Results After excluding patients with incomplete data or follow-up $(n=432)$, we included 373 patients with full data for T-MACS at T0; and 330 who had available samples drawn at 3h. In total $61(15.8 \%)$ patients had AMI and $72(18.7 \%)$ had one or more MACE at 30 days.

Using POC Alere cTnI with T-MACS rule had an area under the receiver operating characteristic curve of 0.915 at T0 and 0.921 at T3. Also, this study has shown that with the Alere POC cTn assay, T-MACS at T0 could have immediately ruled-out AMI in 131 (35.1\%) patients. Of those patients, 3 would have had missed AMI (2.3\%) and 2 (1.5\%) had another MACE within 30 days. With a repeat test at $3 \mathrm{~h}, \mathrm{~T}$ MACS could have ruled-out 110 (33.3\%) patients, missing just one AMI $(0.9 \%)$ and $2(1.8 \%)$ had another MACE within 30 days. In addition, T-MACS could rule-in AMI in 43 $(11.5 \%)$ patents at T0, of which $36(83.7 \%)$ had AMI; and in $48(14.5 \%)$ patients at T3, of which $40(83.3 \%)$ had AMI (figure 1).

Conclusion In conclusion, using T-MACS with the Alere Cardiac Triage 3 POC cTnI assay has insufficient sensitivity to rule-out ACS with one test. It could, however, potentially be used to rule-out ACS when central laboratory assays are unavailable and could be used to enable early rule-in of ACS in the ED.

Conflict of Interest Alere (donation of reagents)

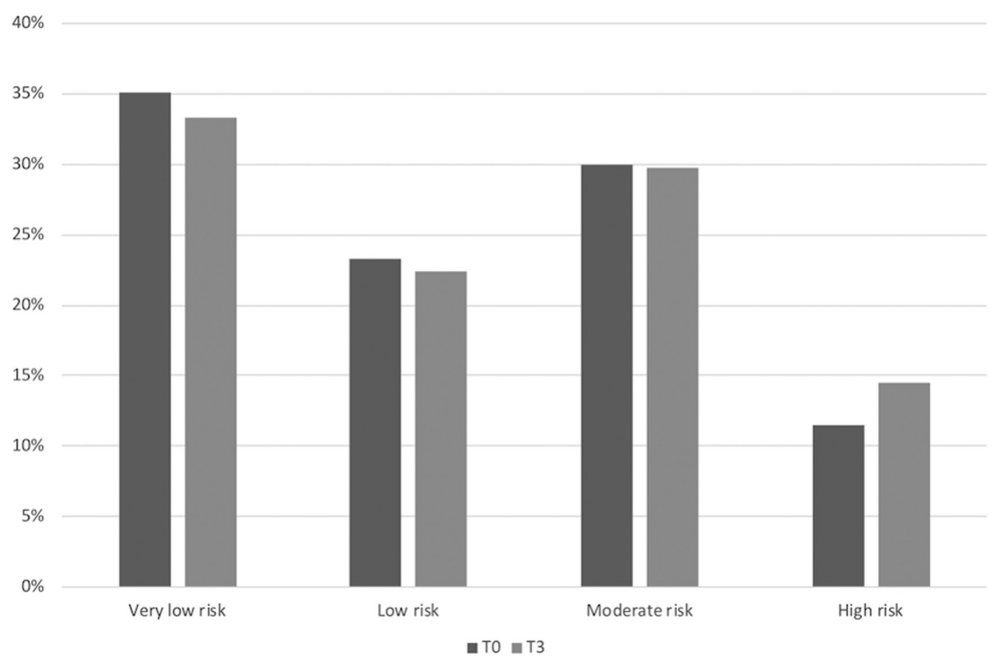

Abstract 156 Figure 1 